\title{
ASSOCIATION ANALYSIS OF $\gamma 2$ SUBUNIT OF $\gamma$-AMINOBUTYRIC ACID TYPE- A RECEPTOR (GABRG2) AND LEUCINE-RICH GLIOMA-INACTIVATED 4 GENE (LGI-4) POLYMORPHISMS WITH IDIOPATHIC EPILEPSIES AND FEBRILE SEIZURES
}

\author{
Gaurav Gupta1, Navneet Kumar², Rakesh Chandra ${ }^{3}$ \\ ${ }_{1}^{1}$ Associate Professor, Department of Medicine, GSVM Medical College, Kanpur, Uttar Pradesh, India. \\ 2 Professor, Department of Neurology, GSVM Medical College, Kanpur, Uttar Pradesh, India. \\ ${ }^{3}$ Ex-Professor, Department of Medicine, GSVM Medical College, Kanpur, Uttar Pradesh, India.
}

\begin{abstract}
BACKGROUND

Epilepsy is the most common neurological disorder affecting 60 million people worldwide and in an estimated $40 \%$ of whom it is genetically determined. Idiopathic epilepsies are those in which genetic aetiology is likely or proven. An alteration of GABA-ergic neurotransmission has been implicated as an aetiologic factor in epileptogenesis, while LGI-4 (Leucine Rich Glioma Inactivated 4) is not homologous to any known ion channel and the mechanism by which it causes epilepsy is unknown. LGI-4 belongs to a super family LRR (Leucine Rich Repeats) proteins and suspected to have a role in peripheral nerve myelination.

The authors aimed to determine whether genetic variations in the GABRG2 and LGI-4 gene confers susceptibility to idiopathic epilepsy and febrile seizures or not.
\end{abstract}

\section{MATERIALS AND METHODS}

In this case control study, samples of 30 cases and 42 controls were taken. Allelic frequencies were expressed as a percentage of the total number of alleles. Genotypes and allelic frequencies for c.588C/T in fifth exon of gene GABRG2 (SNP211037, Asn196Asn) and c.1914GC/AT located in ninth exon of the gene LGI-4 were compared in both groups.

\section{RESULTS}

Association analysis of GABRG-2 polymorphism between CC, CT and TT genotypes statistically showed insignificant result as $\chi^{2}$ value was 0.12 and $p$ value was > 0.05. Association analysis of LGI-4 polymorphism between GC/GC, GC/AT and AT/AT genotypes statistically showed insignificant result as $\chi^{2}$ value was 0.51 and $p$ value was $>0.05$.

\section{CONCLUSION}

It was concluded that GABRG2 and LGI-4 polymorphisms were not the susceptibility factors for idiopathic epilepsies and febrile seizures.

\section{KEY WORDS}

$\gamma$-Aminobutyric Acid Type A Receptor (GABRG2) Polymorphism, Leucine-Rich Glioma-Inactivated 4 Gene (LGI-4) Polymorphism, Idiopathic Epilepsies and Febrile Seizures.

HOW TO CITE THIS ARTICLE: Gupta G, Kumar N, Chandra R. Association analysis of $\gamma 2$ subunit of $\gamma$-aminobutyric acid type- a receptor (GABRG2) and leucine-rich glioma-inactivated 4 gene (LGI-4) polymorphisms with idiopathic epilepsies and febrile seizures. J. Evolution Med. Dent. Sci. 2018;7(39):4282-4285, DOI: 10.14260/jemds/2018/955

\section{BACKGROUND}

Epilepsy is the most common neurological disorder affecting 60 million people worldwide and in an estimated $40 \%$ of population it is genetically determined.(1) Genetic research is crucial in understanding seizure disorders. In general, the epilepsies have multifactorial aetiology with interacting genetic and environmental factors. The most important risk factor for epilepsy is probably genetic heterogenicity and therefore by understanding how genes translate into phenotypes will give important insight into the biology of the epilepsies. Studies in pharmacogenomics use allelic association frequencies to identify genetic variations related to antiepileptic drug response or toxicity.(2)

'Financial or Other Competing Interest': None.

Submission 20-07-2018, Peer Review 11-09-2018,

Acceptance 18-09-2018, Published 24-09-2018.

Corresponding Author:

Dr. Navneet Kumar

7/210-G, Swaroop Nagar,

Kanpur-208002,

Uttar Pradesh, India.

E-mail: neuroindia3@gmail.com

DOI: $10.14260 /$ jemds $/ 2018 / 955$
By understanding the genetic influences on individual variability in drug response may in future give insight to health care practitioners to identify patients at risk of toxicity from certain medications, adjust doses based on genetically determined rates of drug metabolism and select appropriate medications for patients based on their genetic profile.

In general, idiopathic epilepsy arise from genetic abnormalities, leading to alteration of basic neuronal regulation. They comprise of rare mendelian or monogenic epilepsies and more frequent forms, which are familial but they manifest as complex non-mendelian traits. Ion channels are potential candidates to account for the polygenic inheritance of seizure susceptibility. For Idiopathic Generalised Epilepsy (IGE), the estimated concordance among monozygotic and dizygotic twins was estimated to be $76 \%$ and 33\% respectively, which suggests that IGE is a highly specific genetic condition and that genes are involved in its aetiology.(3)

Single nucleotide polymorphisms are markers that provide a new way to study complex gene-associated diseases such as idiopathic epilepsies. An SNP is a single nucleotide variation in genomic DNA and thus different nucleotide variants exist in different members of species. 
Each variant is called an allele. A significantly increased frequency of a variant in people with epilepsy would suggest either that it directly correlates with the risk for epilepsy or that it is located very close to a functional variant on the same chromosome, and very often inherited with the functional variant (linkage disequilibrium).(4)

An alteration of GABAergic neurotransmission has been implicated as an aetiologic factor in epileptogenesis.(5-7) Neuronal inhibition in the mammalian brain occurs largely due to the binding of GABA to heteromeric GABRs. (6-7) GABR, a ligand-gated chloride channel functions as a tetramer consisting of $\alpha, \beta \gamma$, and $\pi$ subunits. Each subunit has several subtypes and the main GABR in the CNS is composed of $\alpha 1$, $\beta 2$ and $\gamma 2$ subunits. The genes encoding GABR subunits represent major candidates for idiopathic generalised epilepsy susceptibility genes because of the widespread distribution of GABRs in the CNS, their ability to produce postsynaptic inhibition and their potential modulation by clinically important anticonvulsant drugs including benzodiazepines and barbiturates.(6) In the past few years, studies done have shown increasing evidence of association of GABRG2 polymorphism with childhood absence epilepsy (CAE), ${ }^{(8)}$ febrile seizures (FS) ${ }^{(8,9)}$ and generalised epilepsy with FS plus (GEFS+). $(8,10)$

LGI-4 (Leucine Rich Glioma Inactivated 4) belongs to a super family LRR (Leucine Rich Repeats) group of proteins.(11) The LGI protein has a distinctive Leucine-Rich Repeat (LRR) motif in its $\mathrm{N}$-terminal end and so called as epilepsy associated EPTP domain. This motif might either function as receptor or interact with the extracellular matrix. The LRR motif consists of repeated $\beta$-strands and $\alpha$-helices connected by loops, building a functional domain that usually serves as a framework for the formation of protein-protein interactions and is present in a large number of proteins with diverse physiological functions. The C-terminal half of the LGI-1 protein consist of seven Epilepsy-Associated Repeats (EARs). EARs/ EPTP are characterised by tandem repeats with a core of about 50 residues that probably folds into a $\beta$ propeller structure.(12) LGI-4 is secreted protein that may play a role in myelination of peripheral nerves. Mutations in the LGI-1/ Epitempin gene has been responsible for causing Autosomal Dominant Lateral Temporal Lobe Epilepsy (ADLTE), a partial epilepsy characterised by the presence of auditory seizures. However, not all the pedigrees with a phenotype consistent with ADLTE show mutations in LGI1/Epitempin gene or have evidence for linkage to the 10q24 locus.(13) Previous studies have shown evidence of association of LGI-4 polymorphism with Benign Familial Infantile Convulsions (BFIC) ${ }^{(9)}$ and childhood absence epilepsy.(14)

In this study, we tested whether genetic variations in the GABRG2 and LGI-4 gene confers susceptibility to idiopathic epilepsy and febrile seizures or not in Indian population.

\section{MATERIALS AND METHODS}

The study was conducted among the patients attending Neuromedicine and Paediatric OPD, LLR and Associated Hospital, GSVM Medical College, Kanpur. Study was conducted over a period of one year. It was a case control type of study. Diagnostic evaluation was made according to classification of ILAE (International League Against Epilepsy) and informed consent was obtained from all participating individuals.

\section{Study Design}

Case control study.

As the study done involved high cost, we have to limit our study to less number of cases and controls with margin of error $>10 \%$. Sample size was taken as per our convenience.

Informed consent was obtained from all the participants.

A case is defined as patients attending the LLR Hospital OPD and who has history of generalised seizures or febrile seizures with no known or suspected aetiology other than possible hereditary predisposition.

Age matched individuals without history of seizures attending the LLR Hospital OPD were taken as controls.

\section{Inclusion Criteria}

- All patients diagnosed with idiopathic epilepsy and febrile convulsions.

- $\quad$ Normal CT/MRI scan.

- Individual family members with a positive history of idiopathic epilepsy and febrile convulsions.

\section{Exclusion Criteria}

- Patients with other types of epilepsy.

- Patients not willing to participate in the study.

- Abnormal CT/ MRI scan.

Blood samples of 30 cases and 42 controls were taken. The distribution of cases with various types of idiopathic epilepsies was tabulated (Table 1).

Extracted DNA of both cases and controls were amplified for GABRG-2 and LG1-4 gene polymorphisms and analysed by PCR followed by restriction analysis, which was done by cleavage at a specific site by restriction enzyme and further analysis was done by PAGE. By analysing banding pattern in PAGE, all the cases and controls into different allelic frequencies were classified. Allelic frequencies were expressed as a percentage of the total number of alleles. Data was analysed using SPSS version 16.0 and $\chi 2$ test was used for statistical analyses.

\begin{tabular}{|c|c|c|}
\hline Type of Idiopathic Epilepsy & Cases & Percentage \\
\hline $\begin{array}{c}\text { Idiopathic generalised tonic- } \\
\text { clonic seizures }\end{array}$ & 8 & $26.67 \%$ \\
\hline Idiopathic focal seizures & 2 & $6.67 \%$ \\
\hline Absence epilepsy & 3 & $10 \%$ \\
\hline Myoclonic astatic epilepsy & 1 & $3.33 \%$ \\
\hline GEFS+ & 1 & $3.33 \%$ \\
\hline JME & 10 & $33.33 \%$ \\
\hline Idiopathic febrile seizures & 5 & $16.67 \%$ \\
\hline \multicolumn{2}{|c|}{ Table 1 } \\
\hline
\end{tabular}

\section{RESULTS}

\section{GABRG2 Polymorphism}

The polymorphism at nucleotide location GCTGAGTGCCAATTA CAATTG CACAA [C/T] TTTCCAATGGATGAACACTCCTGCC (c.588C/T) in fifth exon of gene GABRG2 (SNP211037 Asn196Asn) was studied. By analysing banding pattern in PAGE, all the cases and controls into were classified CC, CT and TT genotypes. 


\begin{tabular}{|c|c|c|c|c|c|}
\hline Genotypes & Cases & Controls & Total (\%) & $\chi^{2}$ & $\begin{array}{c}P \\
\text { value }\end{array}$ \\
\hline $\mathrm{C} / \mathrm{C}$ & 18 & 24 & $42(58.3 \%)$ & \multirow{3}{*}{0.12} & \multirow{3}{*}{0.94} \\
\hline $\mathrm{C} / \mathrm{T}$ & 11 & 16 & $27(37.5 \%)$ & & \\
\hline $\mathrm{T} / \mathrm{T}$ & 1 & 2 & $3(4.2 \%)$ & & \\
\hline Total (\%) & $30(41.7 \%)$ & $42(58.3 \%)$ & \multicolumn{3}{|c|}{$72(100 \%)$} \\
\hline & & Table 2 & & & \\
\hline
\end{tabular}

Association analysis of GABRG-2 polymorphism between CC, CT and TT genotypes using $2 \times 3$ contingency table, degree of freedom 2 and Chi-square test for independence statistically showed insignificant result as $\chi^{2}$ value was 0.12 and $\mathrm{p}$ value was $>0.05$ (Table 2). The odds ratio for individuals with the GABRG2 (SNP211037)-C/C genotype was 1.5 compared with individuals with the GABRG2 (SNP211037)-T/T genotype.

\section{LGI-4 Polymorphism}

The polymorphism at nucleotide location GCGAAGTCGCTGCCTAGGATGGCCA [GC/AT] TGGTCCCTGGC GATGAGCAGTGGCT (c.1914GC/AT) located in ninth exon of the gene LGI-4 was studied. By analysing banding pattern in PAGE, cases and controls were classified into GC/GC, GC/AT and AT/AT genotypes.

\begin{tabular}{|c|c|c|c|c|c|}
\hline Genotypes & Cases & Controls & $\begin{array}{c}\text { Total } \\
(\%)\end{array}$ & $\chi^{2}$ & P value \\
\hline GC/GC & 9 & 15 & $\begin{array}{c}24 \\
(33.3 \%)\end{array}$ & \multirow{3}{*}{0.514} & \multirow{3}{*}{0.77} \\
\hline GC/AT & 15 & 21 & $\begin{array}{c}36 \\
(50.00 \%)\end{array}$ & & \\
\hline $\mathrm{AT} / \mathrm{AT}$ & 6 & 6 & $\begin{array}{c}12 \\
(16.67 \%)\end{array}$ & & \\
\hline Total (\%) & \begin{tabular}{|c|}
30 \\
$(41.6 \%)$ \\
\end{tabular} & $\begin{array}{c}42 \\
(58.3 \%) \\
\end{array}$ & \multicolumn{3}{|c|}{$\begin{array}{c}72 \\
(100 \%)\end{array}$} \\
\hline
\end{tabular}

Association analysis of LGI-4 polymorphism between GC/GC, GC/AT and AT/AT genotypes using $2 \times 3$ contingency table, degree of freedom 2 and Chi-square test for independence statistically showed insignificant result as $\chi^{2}$ value was 0.51 and $p$ value was $>0.05$ (Table 3 ). The odds ratio for individuals with the LGI-4 (c.1914, exon 9) GC/GC genotype was 0.6 when compared to AT/AT genotype.

\section{DISCUSSION}

Chou IC et al found association of $\gamma-2$ subunit of $\gamma$ aminobutyric acid polymorphisms with febrile seizures.(15) They found that children with GABRG2 (SNP211037) C allele has a higher incidence of febrile seizures. A study by Bonani $P$ et al found association mutation of GABR-2 gene with idiopathic generalised epilepsy.(16) In our study, GABRG2 polymorphism is not linked to presence of idiopathic epilepsy and febrile seizures.

LGI-4 belongs to super family LRR proteins. By genomic sequence analysis and radiation hybrid analysis, $\mathrm{Gu}$ et al mapped the LGI-4 gene to chromosome 19q13.11 a chromosomal region linked to Benign Familial Infantile Convulsion (BFIC).(14) The same region showed evidence for a susceptibility gene for idiopathic generalised epilepsy in a genome scan including 130 IGE multiplex families. Wenli $\mathrm{Gu}$ et al showed evidence for LGI-4 polymorphism as a susceptibility gene for childhood absence epilepsy.(14) A genotypic association was found for the c.1914GC/AT polymorphism in CAE patients. In our study, LGI-4 polymorphism is not linked to presence of idiopathic epilepsy and febrile seizures.

\section{CONCLUSION}

Previous studies linked GABRG2 and LGI-4 polymorphisms with idiopathic epilepsies and febrile convulsions, our study did not show this association probably due to difference in race, ethnicity, small size of study population and various limitations of allelic association studies. It was concluded that GABRG2 and LGI-4 polymorphisms were not the susceptibility factors for idiopathic epilepsies and febrile seizures.

\section{ACKNOWLEDGEMENT}

This research was supported by Department of Biosciences and Bioengineering Department, Indian Institute of Technology, Kanpur. We thank BSBE Department, IIT Kanpur who provided insight and expertise that greatly assisted the research.

\section{REFERENCES}

[1] Hausers WA, Annegers JF, Rocca WA. Descriptive epidemiology of epilepsy and contributions of population based studies from Rochester, Minnesota. Mayo Clinic Proc 1996;71(6):576-86.

[2] Ferraro TN, Dlugos DJ, Buono RJ. Challenges and opportunities in the application of pharmacogenetics to antiepileptic drug therapy. Pharmacogenomics 2006;7(1):89-103.

[3] Berkovic SF, Howell RA, Hay DA, et al. Epilepsies in twins: genetics of the major epilepsy syndromes. Ann Neurol 1998;43(4):435-45.

[4] Engel J Jr, Pedley TA. Epilepsy: a comprehensive textbook. Philadelphia, Davis: 2007.

[5] Snodgrass SR. GABA and epilepsy: their complex relationship and the evolution of our understanding. J Child Neurol 1992;7(1):77-86.

[6] Macdonald RL, Olsen RW. GABAA receptor channels. Annu Rev Neurosci 1994;17:569-602.

[7] Olsen RW, Avoli M. GABA and epileptogenesis. Epilepsia 1997;38(4):399-407.

[8] Audenaert D, Schwartz E, Claeys KG, et al. A novel GABRG2 mutation associated with febrile seizures. Neurology 2006;67(4):687-90.

[9] Wang X, Xu M, Du L. Association analysis of gamma2 subunit of gamma-aminobutyric acid (GABA) type A receptor and voltage-gated sodium channel type II alpha-polypeptide gene mutation in southern Chinese children with febrile seizures. J Child Neurol 2007;22(6):714-9.

[10] Harkin LA, Bowser DN, Dibbens LM, et al. Truncation of the GABA(A)-receptor gamma2 subunit in a family with generalized epilepsy with febrile seizures plus. Am J Hum Genet 2002;70(2):530-6.

[11] $\mathrm{Gu} \mathrm{W,} \mathrm{Wevers} \mathrm{A,} \mathrm{Schroder} \mathrm{H,} \mathrm{et} \mathrm{al.} \mathrm{The} \mathrm{LGI1} \mathrm{gene}$ involved in lateral temporal lobe epilepsy belongs to a new subfamily of leucine-rich repeat proteins. FEBS Lett 2002;519(1-3):71-6. 
[12] Staub E, Perez-Tur J, Siebert R, et al. The novel EPTP repeat defines a superfamily of proteins implicated in epileptic disorders. Trends Biochem Sci 2002;27(9):441-4.

[13] Ayerdi-Izquierdo A, Stavrides G, Sellés-Martínez JJ, et al. Genetic analysis of the LGI/Epitempin gene family in sporadic and familial lateral temporal lobe epilepsy. Epilepsy Res 2006;70(2-3):118-26.

[14] Gu W, Sander T, Becker T, et al. Genotype association of exomic LGI4 polymorphisms and childhood absence epilepsy. Neurogenetics 2004;5:41-4.
[15] Chou IC, Peng CT, Huang CC, et al. Association analysis of $\gamma 2$ subunit of $\gamma$-Aminobutyric acid type A receptor polymorphisms with febrile seizures. Pediatric Research 2003;54(1):26-9.

[16] Bonanni P, Malcarne M, Moro F, et al. Generalized epilepsy with febrile seizures plus(GEFS+): clinical spectrum in seven Italian families unrelated to SCN1A, SCN1B and GABRG2 gene mutations. Epilepsia 2004;45(2):149-58. 